\title{
O rgan directed toxicity of halquinol in a repeated dose 28 day oral toxicity study in female rats
}

\author{
R. Swetha, K. J ayakumar, M. Narayanaswamy*, N.B. Shridhar, J agadish Sanganal, Suguna Rao**
}

Departments of Pharmacology and Toxicology, *Physiology and **Veterinary Pathology, Veterinary College, KVAFSU, Hebbal, Bangalore - 560 024, India

Received: 10.4 .2006

Revised: 12.10 .2006

Accepted: 4.1 .2007

Correspondence to: K. Jayakumar E-mail: mail4jk@rediffmail.com

\begin{abstract}
O bjective: The study was undertaken to assess the organ directed toxicity of halquinol in female wistar al bino rats.

Materials and Methods: Halquinol was administered orally by gavage at the dose of 0 (control), 150 (low), 450 (intermediate) and 1000 (high) mg/kg body weight daily for a period of 28 days to four groups of rats $(n=6)$. Each rat was weighed at the beginning and at weekly intervals thereafter till the end of the study. Blood and serum samples were analyzed on Day 0,14 and 28 for haematological parameters viz, Hb, Hct, TEC, MCV, $\mathrm{MCH}, \mathrm{MCHC}, \mathrm{TLC}$ and DLC and serum biochemical parameters viz, ALP, ALT, AST, TSP, TA, BU N and Creatinine respectively. At the end of the study organs were weighed and organ to body weight ratios were calculated. The representative tissue samples were processed for histopathological examination.

Results: The body weight in the intermediate and high dose group was significantly $(P<0.01)$ lower than the control group. The $\mathrm{Hb}, \mathrm{TEC}$ and $\mathrm{MCHC}$ decreased significantly $(P<0.01)$ whereas MCV increased significantly $(P<0.01)$ in high dose group. Serum biochemical parameters viz, ALP, ALT, BUN and creatinine significantly $(P<0.01)$ increased in intermediate and high dose groups. O rgan to body weight ratio in case of liver and kidney was also significantly $(P<0.01)$ higher in high dose group. Histopathology of liver and kidney from intermediate and high dose groups revealed marked pathological alterations.
\end{abstract}

Conclusion: From the present study it was concluded that at intermediate and high dose, halquinol was hepatotoxic and nephrotoxic in female rats.

KEY WORDS: Biochemistry, halquinol, hepatotoxicity, histopathology, nephrotoxicity.
Quinoline derivatives were introduced way back in 1960s for the treatment of amoebic dysentery in man. The use of an oxyquinoline derivative iodochlorhydroxyquinoline was banned since prolonged usage caused a neurological syndrome subacute myelo-optic neuropathy (SMON) in Japanese people. However, recently evidence has accumulated to suggest that metal chelator iodochlorhydroxyquinoline has its potential usage in control of oxidative damage in parkinson's, alzheimer's, amylotrophic lateral sclerosis and other neurodegenerative diseases. ${ }^{[1]}$ Halquinol, a related drug having antibacterial, antifungal and antiprotozoal activity is still being used in India to overcome common challenges of modern poultry and swine farming like microbial infections and growth promotion aspects. Neurotoxicity is reported with halquinol. ${ }^{[2]}$ However, the details of toxicity of halquinol are scanty. Hence the present study was undertaken to assess the organ directed toxicity of halquinol in a repeated dose 28-day oral toxicity study in female rats.

\section{Materials and Methods}

Halquinol is a mixture obtained by chlorinating quinolin8-ol. It contains not less than 57 per cent and not more than 74 per cent of 5, 7-dichloroquinolin-8-ol, not less than 23 per cent and not more than 40 per cent of 5-chloroquinolin-8-ol and not more than 4 per cent of 7 -chloroquinolin-8-ol and the total content of the three components is not less than 95 per cent and not more than 105 per cent. ${ }^{[3]}$ The oxyquinoline derivative halquinol (HALQUINOL BP 80. 98\% W/W, manufactured and supplied by M/s. Vetcare, Bangalore) was used in the present study. The study was conducted as per OECD test guidelines ${ }^{[4]}$ for repeated dose 28-day Oral Toxicity Study in Rodents. Female wistar albino rats weighing $110-115 \mathrm{~g}$ and aged between six to seven weeks were procured from the Laboratory Animal Facility of Veterinary College, Hebbal, Bangalore. After acclimatization for a week, animals were grouped into four groups and each 
group consisted of six rats. They were maintained under standard laboratory hygienic conditions, providing standard laboratory animal feed and water ad libitum. The approval of the Institutional Animal Ethics Committee was obtained prior to start of the experiment.

Group I served as control. Halquinol suspended in tragacanth mucilage (BP 1973) was administered by oral gavage as a single dose using a stomach tube, at the dose of $150 \mathrm{mg} / \mathrm{kg}$ body weight (Low dose) to Group II, $450 \mathrm{mg} / \mathrm{kg}$ body weight (Mid dose) to Group III and $1000 \mathrm{mg} / \mathrm{kg}$ body weight (high dose) to Group IV daily for a period of 28 days. The doses were selected on the basis of acute toxicity study and limit test where the highest dose was chosen with the aim of inducing toxic effects but not death or severe suffering. Thereafter, a descending sequence of dose level was selected with an aim to demonstrate a dose related response and no-observed adverse effects at the lowest dose level (NOAEL). Each rat was weighed at the beginning of the study and at weekly intervals thereafter till the end of the study. Exposure to halquinol in poultry and swine farming depends on the stage of life. Halquinol to weaning piglets and growers is given at the rate of $30-60 \mathrm{~g} /$ tonne of feed whereas to finishers it is $30 \mathrm{~g} /$ tonne of feed. For chronic scouring, halquinol is used at the rate of $600 \mathrm{~g} /$ tonne of feed for up to 7 days. In broilers, it is mixed in the feed at the concentrations of 30-60 $\mathrm{g} /$ tonne and in layers from 9 to 16 weeks of age halquinol is given at the rate of $30 \mathrm{~g} / \mathrm{tonne}$ of feed and 30-60 g/tonne of feed in the later laying cycle.

General observations were made at least twice daily for signs of toxicity. Blood samples were collected by puncturing the retro orbital plexus using microhaematocrit capillary tubes on Day 0, 14 and 28 and subjected for analysis of haematological parameters viz, haemoglobin $(\mathrm{Hb})$, haematocrit (Hct), total erythrocyte count (TEC), mean corpuscular volume (MCV), mean corpuscular haemoglobin (MCH), mean corpuscular haemoglobin concentration (MCHC), total leukocyte count (TLC) and differential leukocyte count (DLC) and serum biochemical parameters viz, alkaline phosphatase (ALP), alanine aminotransferase (ALT), aspartate aminotransferase (AST), total serum protein (TSP), total albumin (TA), blood urea nitrogen (BUN) and creatinine (Creat). Disodium EDTA was used as an anticoagulant and the haematological parameters were determined following standard methods. ${ }^{\left[{ }_{1}\right]}$ The biochemical parameters were analyzed using ARTOS Semi-Automatic Biochemical Analyzer. The commercially available diagnostic kits from M/s. Swemed diagnostics, Bangalore, were employed in the estimation of the above parameters. After overnight fasting, the rats were weighed and sacrificed on Day 28 under ether anesthesia and the necropsy was conducted on each carcass to note down gross pathological changes. Organs namely liver, kidney, heart, lung and spleen were collected and weighed carefully on an analytical balance and weight of each organ recorded. Organ to body weight ratio was calculated. The representative tissue samples were collected in neutral buffered formalin and processed for histopathology by cutting sections of 5 micron thickness and staining with haematoxylin and eosin. ${ }^{|6|}$

The data was expressed as mean \pm standard error of mean (SEM). The data was analyzed statistically by One-way ANOVA followed by Dunnett's post test using GraphPad Prism software, version $4.01 .^{[7]}$

\section{Results}

The observed clinical signs of toxicity in the present study were burrowing behavior soon after administration of halquinol in intermediate and high dose group. The high dose group showed gradual loss of body weight and initiation of diarrhoea after day 14. Day 20 onwards-severe diarrhoea, yellow urine, malena, dullness, depression, emaciation and hind leg weakness resulting in altered gait were observed. The above clinical signs were not evident in other groups given lower doses except mild diarrhoea in the intermediate dose group after $20^{\text {th }}$ day of administration of halquinol. The body weight in the Group III and Group IV was significantly $(\mathrm{P}<0.01)$ lower than the control group value [Table 1]. The Hb, TEC and MCHC in Group IV was significantly ( $P<0.01)$ lower than the control group value whereas the MCV in Group IV was significantly $(P<0.01)$ higher than the control group value [Table 2]. There was no significant $(P>0.05)$ increase in Hct, MCH, TLC and DLC values in all the groups administered halquinol when compared to control. There was also significant $(P<0.01)$ increase in ALT, ALP, BUN and creatinine concentration in Group III and Group IV [Table 3] but there was no significant $(P>0.05)$

\section{Table 1}

Effect of Halquinol on body weight $(g)$ in female rats in 28 day oral toxicity study

\begin{tabular}{|c|c|c|c|c|c|c|}
\hline Group/day & 0 & 7 & 14 & 21 & 28 & $\%$ change on $28^{\text {th }}$ day \\
\hline \multicolumn{7}{|l|}{ Group I } \\
\hline Control & $101.667 \pm 1.054$ & $141.667 \pm 2.108$ & $182.500 \pm 2.814$ & $215.833 \pm 2.386$ & $215.833 \pm 2.386$ & $+112 \pm 2.8$ \\
\hline \multicolumn{7}{|l|}{ Group II } \\
\hline $150 \mathrm{mg} / \mathrm{kg}$ & $103.333 \pm 1.667$ & $140.000 \pm 1.826$ & $170.833 \pm 2.007$ & $181.667 \pm 2.108$ & $210.833 \pm 2.386$ & $+104 \pm 2.4$ \\
\hline \multicolumn{7}{|l|}{ Group III } \\
\hline $450 \mathrm{mg} / \mathrm{kg}$ & $103.333 \pm 1.667$ & $123.333 \pm 1.667^{* *}$ & $149.167 \pm 2.386^{* *}$ & $135.000 \pm 2.582^{* *}$ & $157.000 \pm 3.819^{* *}$ & $+53 \pm 4.3^{* *}$ \\
\hline \multicolumn{7}{|l|}{ Group IV } \\
\hline $1000 \mathrm{mg} / \mathrm{kg}$ & $100.833 \pm 0.833$ & $112.500 \pm 1.708^{* *}$ & $119.167 \pm 1.536^{* *}$ & $121.667 \pm 1.667^{* *}$ & $125.833 \pm 2.386^{* *}$ & $+25 \pm 2.2^{* *}$ \\
\hline \multicolumn{7}{|c|}{ One-way ANOVA } \\
\hline $\mathrm{F}$ & 0.85 & 58 & 150 & 380 & 240 & 190 \\
\hline$P$ & $>0.05$ & $<0.01$ & $<0.01$ & $<0.01$ & $<0.01$ & $<0.01$ \\
\hline
\end{tabular}

Values are given as mean \pm S.E.M.; $n=6$ in each group; ${ }^{*} P<0.05$ and ${ }^{* *} P<0.01$ as compared to control; $d f=3,20$ 


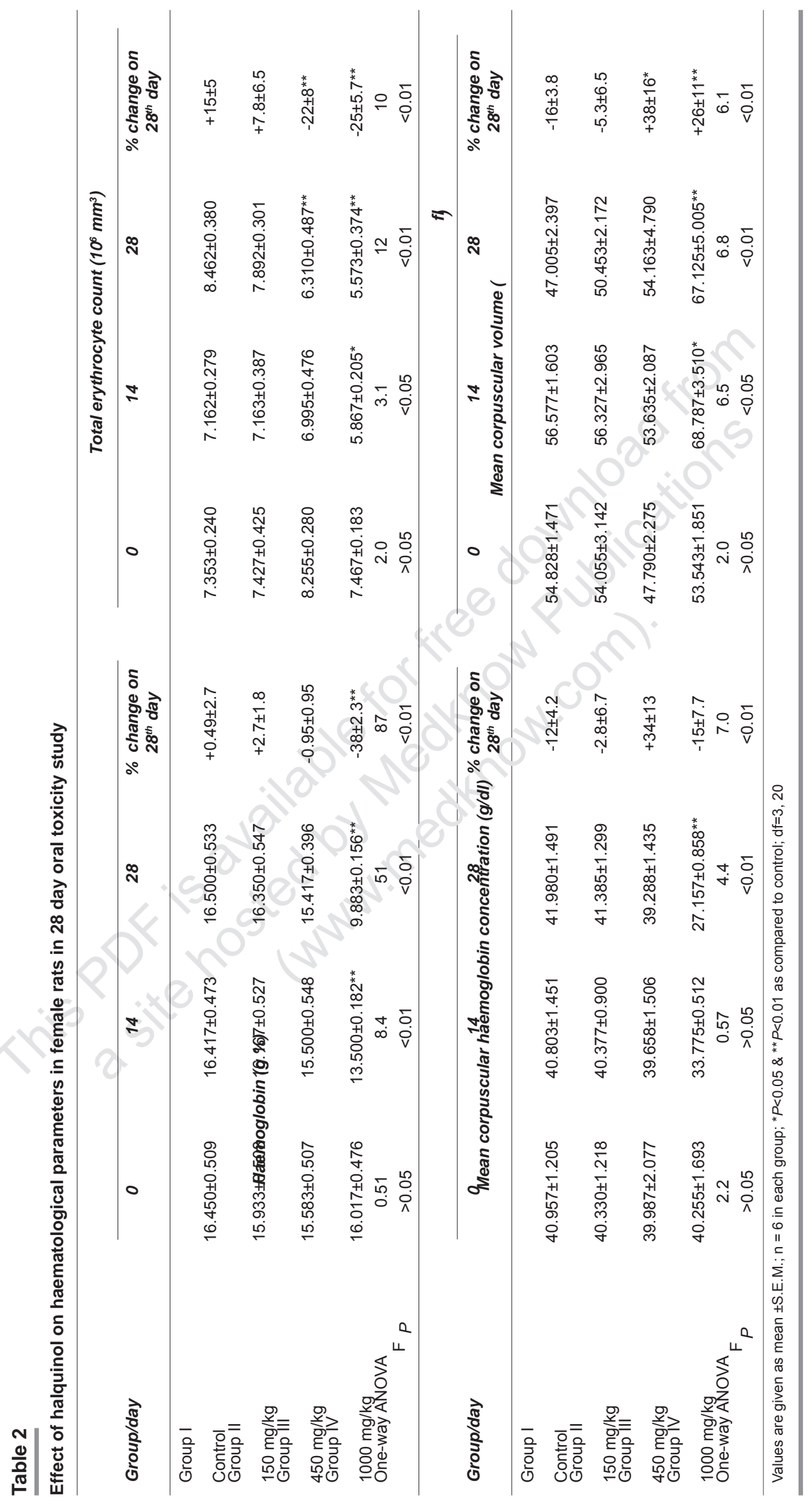




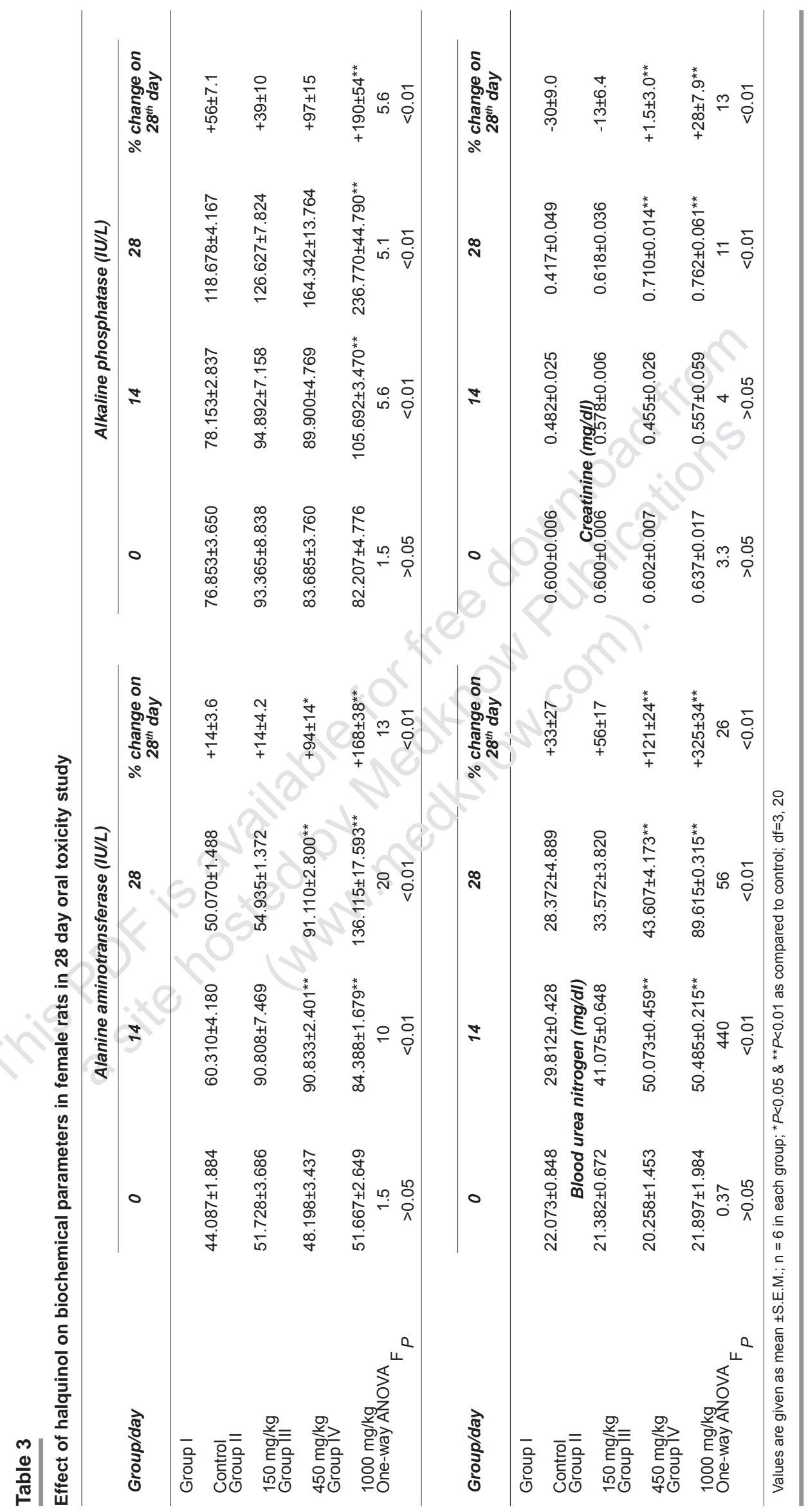


increase in AST, TSP and TA concentration. There was significant $(\mathrm{P}<0.01)$ increase in liver to body weight ratio and kidney to body weight ratio in Group III and Group IV [Table 4].

At autopsy, rats in Group III and Group IV showed generalized paleness of mucous membranes and all visceral organs. Animals of the treated groups did not show any gross pathological lesions in any of the organs except for catarrhal enteritis in Group III and Group IV. Microscopically liver from rats of high dose group showed congestion of sinusoidal spaces, hydropic degeneration with granular eosinophilic cytoplasm of hepatocytes and multiple areas of necrosis [Figure 1]. The lesions in kidney microscopically were cystic dilatation of collecting tubules lined by flattened epithelial cells and necrosis of lining epithelium of the kidney tubules. Some renal tubules showed vacuolar degeneration and necrosis of the lining epithelial cells [Figures 2 and 3]. Kidney tubules also showed retention of urine. Microscopically intestines revealed mainly catarrhal inflammation characterized by degeneration and necrosis of tips of intestinal villi, increased goblet cell activity, mild haemorrhage and infiltration of inflammatory cells in lamina propria and widening of lymphoid component in large intestine. Histopathology of rats from intermediate dose group showed similar lesions in liver and kidney but in lesser intensity i.e., microscopically liver showed loss of normal architecture with vacuolar degeneration of hepatocytes and the microscopic lesion in kidney was cystic dilatation of collecting tubules with mild vacuolar degeneration of tubular epithelial cells of renal tubules in some of the areas. Section of intestine also showed catarrhal enteritis with moderate increase in goblet cell activity, loss of intestinal villi, congestion, mild haemorrhages and infiltration of inflammatory cells in lamina propria. Widening of lymphoid component in sub mucosal region was also observed in large intestine

\section{Discussion}

The body weight of high dose and intermediate dose group was significantly $(\mathrm{P}<0.01)$ lower than the control group value. Low dose group showed normal weight gains and exhibited no toxic effects. This finding is similar to the finding reported by Heseltine and Campbell, ${ }^{[8]}$ who reported normal weight gains with no toxic effects in male and female rats given halquinol orally at doses of $0.2 \mathrm{~g} / \mathrm{kg}$ daily for 14 days and $0.6 \mathrm{~g} / \mathrm{kg}$ daily for three days. Ezzedeen and his coworkers ${ }^{[9]}$ reported a weight loss of $15.3 \pm 2.7 \%$ observed over the 28 day topical treatment of clioquinol in five dogs.

The Hb, TEC and MCHC decreased significantly $(P<0.01)$ whereas MCV increased significantly $(\mathrm{P}<0.01)$ in high dose group. The present findings of reduced erythrocyte counts and haemoglobin concentrations are in agreement to Hoover and Carlton, ${ }^{[10]}$ who reported reduced erythrocyte counts and haemoglobin concentrations in Beagle dogs treated with clioquinol for a period of 100 days. The observed fall in haemoglobin concentrations and erythrocyte counts in high dose group might have occurred because of acute loss of blood through faeces (melena). This correlated with the gross pathological change of generalized paleness of all mucous membranes and visceral organs of the body. The observed increase in MCV and decrease in MCHC is suggestive of macrocytic hypochromic anaemia which might have occurred
Figure 1: Section of liver from a female rat administered with high dose of halquinol showing swollen hepatocytes with granular and vacuolar appearance of cytoplasm (line arrow) and infiltration of inflammatory cells in the parenchyma (solid arrow) (H\&E, 500x)

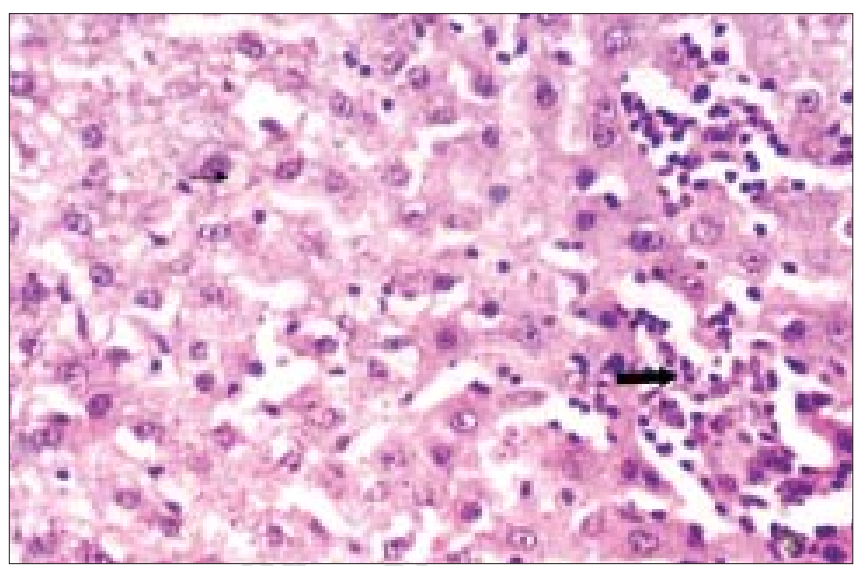

Figure 2: Section of kidney from a female rat administered with high dose of halquinol showing cystic dilatation of collecting tubules (solid arrow) and necrosis of lining epithelium of the kidney tubules (line arrow) (H\&E, 500x)

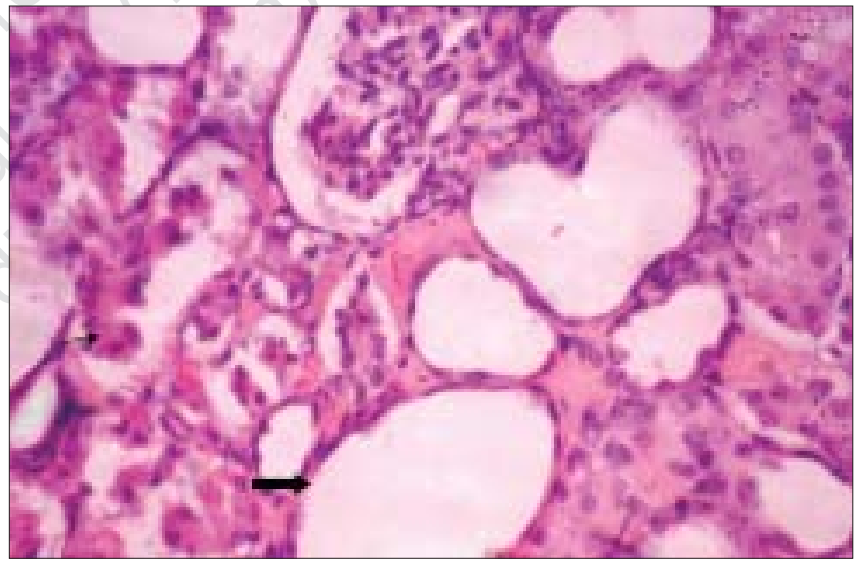

Figure 3: Section of kidney from a female rat administered with high dose of halquinol showing vacuolar degeneration (solid arrow) and necrosis of kidney tubules (line arrow) (H\&E, 500×)

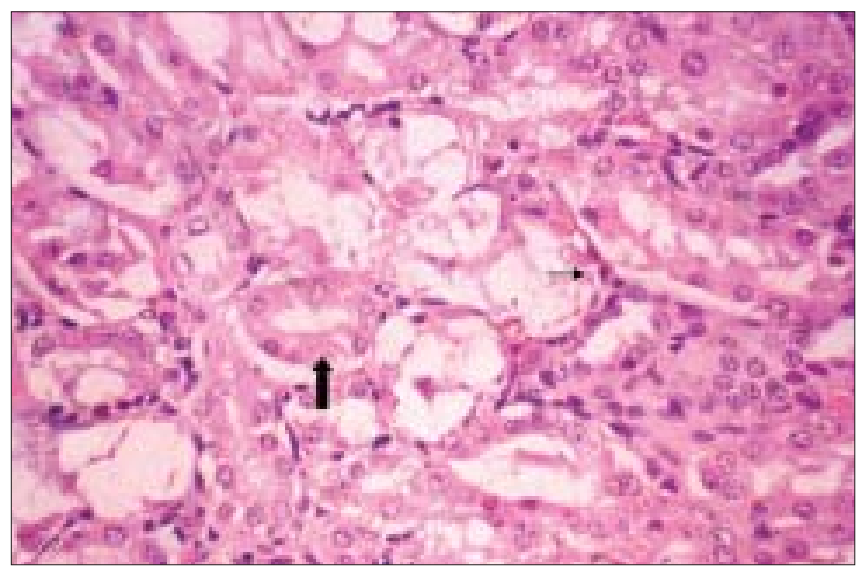


Table 4

Effect of Halquinol on organ to body weight ratio (\%)in female rats in 28 day oral toxicity study

\begin{tabular}{|c|c|c|c|c|c|}
\hline Group/organ & Liver & Kidney & Spleen & Heart & Lungs \\
\hline \multicolumn{6}{|l|}{ Group I } \\
\hline Control & $4.293 \pm 0.168$ & $0.707 \pm 0.005$ & $0.460 \pm 0.023$ & $0.302 \pm 0.006$ & $0.847 \pm 0.028$ \\
\hline \multicolumn{6}{|l|}{ Group II } \\
\hline $150 \mathrm{mg} / \mathrm{kg}$ & $4.000 \pm 0.215$ & $0.733 \pm 0.024$ & $0.425 \pm 0.004$ & $0.285 \pm 0.011$ & $0.797 \pm 0.021$ \\
\hline \multicolumn{6}{|l|}{ Group III } \\
\hline $450 \mathrm{mg} / \mathrm{kg}$ & $5.833 \pm 0.224^{\star *}$ & $1.405 \pm 0.012^{* *}$ & $0.382 \pm 0.012$ & $0.370 \pm 0.004$ & $0.898 \pm 0.031$ \\
\hline \multicolumn{6}{|l|}{ Group IV } \\
\hline 1000 mg/kg & $8.555 \pm 0.331^{* *}$ & $1.600 \pm 0.068^{* *}$ & $0.425 \pm 0.006$ & $0.470 \pm 0.010$ & $0.968 \pm 0.038$ \\
\hline \multirow{2}{*}{ One-way ANOVA } & 74 & 160 & 5.7 & 10 & 6.0 \\
\hline & $<0.01$ & $<0.01$ & $>0.05$ & $>0.05$ & $>0.05$ \\
\hline
\end{tabular}

Values are given as mean \pm S.E.M.; $n=6$ in each group; ${ }^{*} P<0.05 \&{ }^{* *} P<0.01$ as compared to control; $d f=3,20$

because of acute loss of blood through faeces (melena).

High and intermediate dose exerted toxic effect on the liver and the toxic effect on high dose group was more severe compared to that of intermediate dose group, which correlated well with the histopathologic lesions in liver and increase in biochemical parameters ALT and ALP. Halquinol at high dose and intermediate dose also has toxic effect on the kidney. The toxic effect in high dose group is more severe compared to that of intermediate dose group, which correlated well with the histopathology of kidney showing severe damage in the high dose group and milder degree of damage in the intermediate dose group and increase in BUN and creatinine concentration in serum.

The pathological findings in the kidneys were not in accordance to the findings of Heseltine and Campbell ${ }^{[8]}$ who did not find any pathological changes in kidneys in rats fed chlorhydroxyquinoline $0.3 \mathrm{~g} / \mathrm{kg}$ daily for the test period of 3 days. But the present pathological finding in kidney are in agreement to Pashov ${ }^{[11]}$ who has accounted the acute and subchronic toxicity of two hydroxyquinoline derivatives in combination (The Bulgarian preparation Enteroquin which is a mixture of broxyquinoline (INN) and broxaldin (INN) in the ratio $5: 1)$ in which he has reported that oral administration of 0.1 $\mathrm{g} / \mathrm{kg}$ body weight to rats for 32 consecutive days produced no histological changes in internal organs, but $0.24 \mathrm{~g} / \mathrm{kg}$ caused kidney degeneration.

There was significant $(\mathrm{P}<0.01)$ increase in organ to body weight ratio in case of liver which may be due to fatty changes in the liver and also due to diarrhoea and dehydration. Similar increase in relative liver weight due to fatty change, diarrhoea and dehydration was observed in broiler chicks fed diet containing ochratoxin $\mathrm{A} .{ }^{[12]}$ There was also significant $(\mathrm{P}<0.01)$ increase in kidney to body weight ratio, which may be due to cystic dilatation and retention of urine in tubules and also due to diarrhea and dehydration. Similar increase in relative kidney weight due to cystic dilatation and retention of urine was observed in newborn rats with partial unilateral ureteric obstruction. ${ }^{13 \mid}$

Thus from the present study it was concluded that halquinol at high and intermediate dose is hepatotoxic and nephrotoxic in female rats.

\section{References}

1. Barnham KJ, Masters CL, Bush Al. Neurodegenerative diseases and oxidative stress. Nat Rev Drug Discov 2004;3:205-14.

2. Hanson 0 , Herxheimer $O$. Neuropathy and optic atrophy associted with halquinol. Lancet 1981;1:450.

3. Anonymous. British Pharmacopoeia. Her Majesty's Stationery Office: London; 1980. p. 218-9.

4. Anonymous. OECD Test guidelines 425. Organization for Economic cooperation and development, Paris; 2001.

5. Jain NC. Schalm's Veterinary Haematology. $4^{\text {th }}$ ed. KM Verghese Co: New Delhi; 1990.

6. Luna LG. Manual of histopathological staining methods of the armed forces institute of pathology. $3^{\text {rd }}$ ed. McGraw Hill Book Co: New York; 1968.

7. GraphPad Prism, Version 4.01 for Windows, GraphPad Software Inc: San Diego, California, USA; 2004.

8. Heseltine WW, Campbell PJ. Laboratory studies on chlorhydroxyquinoline. J Trop Med Hyg 1960;63:163-5.

9. Ezzedeen FW, Stohs SJ, Kilzer KL, Makoid MC, Ezzedeen NW. Percutaneous absorption and disposition of iodochlorhydroxyquin in dogs. J Pharm Sci 1984;73:1369-72.

10. Hoover DM, Carlton WW. The subacute neurotoxicity of excess pyridoxine $\mathrm{HCl}$ and clioquinol (5-chloro-7-iodo-8-hydroxyquinoline) in beagle dogs. II. Pathology. Vet Pathol 1981;18:757-68.

11. Pashov D. Acute and subchronic toxicity of 8-hydroxyquinolone derivatives in combined application. Vet Med Nauki 1979;16:81-7.

12. Manning $R Q$, Wyatt RD. Toxicity of Aspergillus ochraceus contaminated wheat and different chemical forms of ochratoxin A in broiler chicks. Poult Sci 1984;63:45865.

13. Wen JG, Frokiaer J, Zhao JB, Ringgaard S, Jorgensen TM, Djurhuus JC. Severe partial ureteric obstruction in newborn rats can produce renal dysplasia. BJU Int 2002;89:740-5 